\title{
Congenital Left Ventricular Diverticulum Repair in an Adult with Dual Patch Technique
}

\author{
Javier Mejia ${ }^{1}$, Stephen Spindel $^{2}$, Amit Pawale $^{3}$, Shinobu Itagaki $^{3}$, and Percy Boateng ${ }^{2}$ \\ ${ }^{1}$ Mount Sinai Hospital \\ ${ }^{2}$ The Mount Sinai Hospital \\ ${ }^{3}$ Mount Sinai Medical Center
}

May 6, 2020

\begin{abstract}
Rupture of a congenital left ventricular diverticulum (CLVD), a rare anatomical anomaly, is a catastrophic event, with potential fatal consequences. Repair techniques documented in the literature include primary closure and single patch closure. We describe a case of a 57-year-old woman with symptomatic anterolateral CLVD. Our approach involves a linear incision through the epicardial surface of the diverticulum with exclusion of the cavity, and restoration of normal ventricular geometry via a two patch technique.
\end{abstract}

\section{Introduction}

Congenital left ventricular diverticulum (CLVD) consists of an outpouching of endocardium, myocardium, and pericardium, which often presents as a projection from the ventricular free wall, with a narrow neck connecting the cavity to the ventricle proper [1]. Due to its composition of normal cardiac tissue, as compared to a congenital left ventricular aneurysm (CLVA), the diverticulum contracts synchronously with the remainder of the ventricle. Thromboembolic events, ventricular arrhythmias, heart failure, and free wall rupture have been documented to occur. Due to its low overall prevalence between $0.02 \%$ to $0.76 \%[2,3,4]$, and variability in presentation, a standardized treatment paradigm has yet to be delineated. We report the successful repair of a congenital left ventricular diverticulum using a two patch technique.

\section{Technique}

A healthy 57-year-old female was evaluated for frequent episodes of atypical chest pain, which revealed a CLVD. She had no history of myocardial infarctions, mediastinal infections, or trauma. A computed tomography angiography demonstrated an anterolateral left ventricular outpouching measuring $4.9 \times 3.8 \mathrm{x}$ $1.6 \mathrm{~cm}$, accompanied by a narrow neck with areas of thick and thin wall containing trabeculations. A cardiac magnetic resonance imaging study confirmed similar dimensions and noted the walls to be thinned in focal regions (Fig 2). Coronary angiogram showed normal coronary arteries.

Intraoperative transesophageal echocardiogram showed blood flow into the CLVD. Through a median sternotomy, the patient was placed on cardiopulmonary bypass with ascending aortic arterial and right atrial venous cannulation. The heart was arrested and the left ventricle inspected. A $5 \times 4 \mathrm{~cm}$ area of protrusion with thinned myocardium was noted on the anterolateral aspect of the left ventricle (Fig 1A). The thinned area of tissue was opened through a $5 \mathrm{~cm}$ linear incision (Fig 1B). The discrete neck communicating with 
the left ventricular wall measured $3 \mathrm{~cm}$, allowing flow between the CLVD and the left ventricle. Multiple pledgeted 4-0 polypropylene horizontal mattress sutures were passed from outside-to-in through the rim of healthy appearing myocardium just above and around the diverticular neck. These were then each passed through the perimeter of a circular $6 \times 4 \mathrm{~cm}$ Hemashield (Maquet Holding, Rastatt, Germany) patch that laid on top and flush with the diverticular neck, and subsequently tied (Fig 1C). Care was taken to inspect and avoid the subvalvular mitral apparatus. The patch sealed off the CLVD from the left ventricular cavity while avoiding compromising the true ventricular lumen (Fig 1D), its geometry, or reducing the intraventricular volume.

A second set of pledgeted 4-0 polypropylene sutures were placed from inside the CLVD in a horizontal mattress fashion, circumferentially around the linear incision through the wall of the diverticulum. These sutures were taken more proximal to the epicardial surface, above the level of the first patch placed near the diverticular neck, encircling the incision into the diverticulum (Fig 1E). The incision into the cavity was then oversewn with two running 4-0 polypropylene sutures, with a pledget on either side of the incision, ensuring appropriate approximation, but with care to apply minimal tension to either free edge, limiting how tightly they were brought together (Fig $1 \mathrm{~F})$.

A second circular Hemashield patch $7 \mathrm{~cm}$ in diameter was then fashioned. Half of the adjacent epicardial mattress sutures were then passed through this patch. The patch was subsequently lowered and these adjacent sutures were securely tied, leading to half the patch being secured to the surface of the epicardium. BioGlue (CryoLife Inc, Georgia, United States) was applied to this pocket of space between the epicardium and the Hemashield patch, with the other half of the remaining adjacent sutures then passed through the patch and tied securely once the glue had been allowed to settle (Fig 1G).

The cross clamp was removed and the patient weaned off bypass. Transesophageal echocardiogram showed good biventricular function without a compromise in ventricular lumen, with no communication, and complete exclusion of the diverticular cavity. Her post-operative course was uneventful.

\section{Comment}

CLVD often represent novel territory with regards to management and, at times, diagnosis for most heart teams. Not only is the prevalence fairly low, ubiquitously lower than $1 \%$ as previously described, but additionally the natural history of the disease is not well delineated. There are two distinct pathologies that are clearly described in the literature, with CLVD representing an outpouching from the ventricle, with synchronous contraction, and histologically similar to that of the ventricular wall from which it protrudes. Congenital left ventricular aneurysm (CLVA) represents the other end of the spectrum, whereby the outpouching is dyskinetic or altogether akinetic, and histologically composed of fibrous tissue, dissimilar to that of the ventricular wall [5]. Despite the dearth of epidemiological data, CLVD are not clinically silent, with reports of important cardiac complications such as thromboembolism, ventricular arrhythmias, and myocardial ischemia. Additionally, catastrophic CLVD rupture has been described, leading to sudden death $[6]$.

Due to the rarity of CLVD, there is little guidance for the clinician regarding appropriate treatment paradigms, be it a surgical or conservative approach. In a review of 809 patients published since 1816 with either CLVD or CLVA, $4.5 \%$ of patients with CLVD were found to have an episode of rupture, and a $5.0 \%$ reported rate of cardiac death. Other significant complications included ventricular tachycardia/fibrillation in $13.1 \%$, embolic events $3.6 \%$, and syncope $5.1 \%$ [7].

Despite the existence of a concise review of the cases reported to date, there is little in the way of a detailed surgical approach. Some authors have reported closure of the diverticulum neck with a patch, with surgical glue closure and plication with aneurysmorrhaphy [8]. Other authors have described their technique as suture reapproximation in a double layered fashion with the use of felt [9], while some describe removal of the outpouching cavity and closure with an in-situ patch [10].

Due to the catastrophic events associated with CLVD, our institution prefers surgical management for 
patients with symptomatic CLVD. The first patch of our two patch technique helps retain the native geometry of the left ventricle. Meanwhile, linear closure of the CLVD may cause a reduction of the ventricular diameter and distortion of contractile force vectors. The addition of a second patch to the ventricular epicardial surface in our approach decreases early postoperative bleeding and should minimize long term aneurysm formation and rupture. Further study is necessary to determine the benefits of this technique.

\section{Disclosure}

This manuscript has not been published elsewhere. No conflicts of interest for any of the authors.

\section{References}

1. Ohlow M, -A, Congenital Left Ventricular Aneurysms and Diverticula: Definition, Pathophysiology, Clinical Relevance and Treatment. Cardiology 2006;106:63-72

2. Albrecht G: Morphology and formal etiology of congenital heart diverticula. Zentralbl Allg Pathol 1972;116:42-47.

3. (Cardiology Images 12)Ohlow M.-A., Secknus M. A., Geller J. C., von Korn H., Lauer B. Prevalence and outcome of congenital left ventricular aneurysms and diverticula in an adult population. Cardiology. 2009;112(4):287-293. doi: 10.1159/000159122.

4. (Cardiology Images 18) Albrecht G. Beirtrag zur Morphologie und formalin Genese kongeintaler Divertkil. Zentralbl Allg Pathol. 1972;116(1):42-47.

5. Halpern L, Garabedian C, Worrall NK. Congenital Ventricular Diverticulum or Aneurysm: A Difficult Diagnosis to Make. Case Rep Cardiol. 2018;2018:5839432. Published 2018 Nov 11. doi:10.1155/2018/5839432

6. S. Yalonetsky, Y. Agmon, J. Lessick. Contrast echocardiographic imaging of left ventricular diverticulum in adult patients. J Am Soc Echocardiogr, 20 (2006), pp. e1-e3

7. Ohlow MA, von Korn H, Lauer B. Characteristics and outcome of congenital left ventricular aneurysm and diverticulum: Analysis of 809 cases published since 1816. International Journal of Cardiology. 2015 Apr;185:34-45. DOI: 10.1016/j.ijcard.2015.03.050.

8. Jung JC, Oh HC, Kim KH. Isolated Congenital Left Ventricular Diverticulum in Adults. Korean J Thorac Cardiovasc Surg. 2015;48(5):355-358. doi:10.5090/kjtcs.2015.48.5.355

9. Scardigno AD, Chieppa DRR, Deluca G, Carbonara V. Isolated Congenital Left Ventricular Diverticulum: A Case Report and Differential Diagnosis. J Cardiovasc Echogr. 2016;26(1):19-21. doi:10.4103/22114122.178467

10. Manabu Yamasaki, Naoto Kawamatsu, Kunihiko Yoshino, Kohei Abe, Hiroyasu Misumi, Congenital Left Ventricular Diverticulum Complicated by Ventricular Fibrillation, The Annals of Thoracic Surgery, Volume 104, Issue 3, 2017, Pages e271-e273, ISSN 0003-4975, https://doi.org/10.1016/j.athoracsur.2017.03.029. 


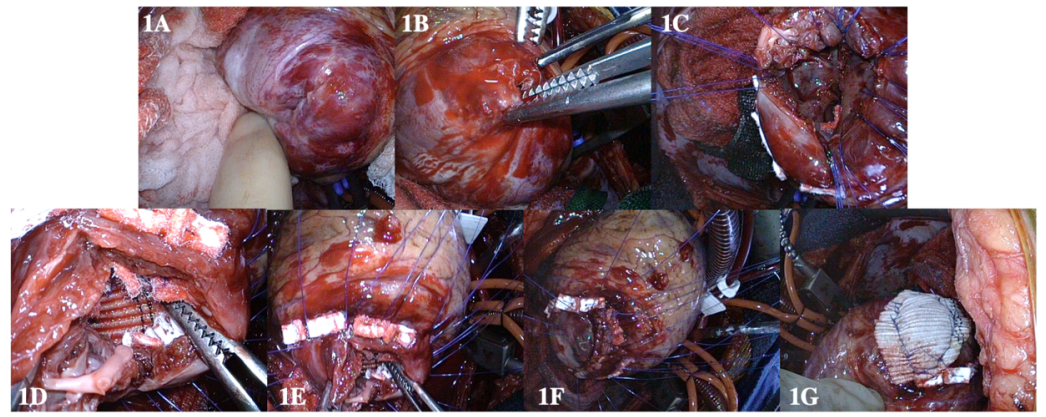

Figure 1A - 1G: Intraoperative surgical repair technique of CLVD by sharp incision, pledgeted mattress sutures, and a dual patch technique.
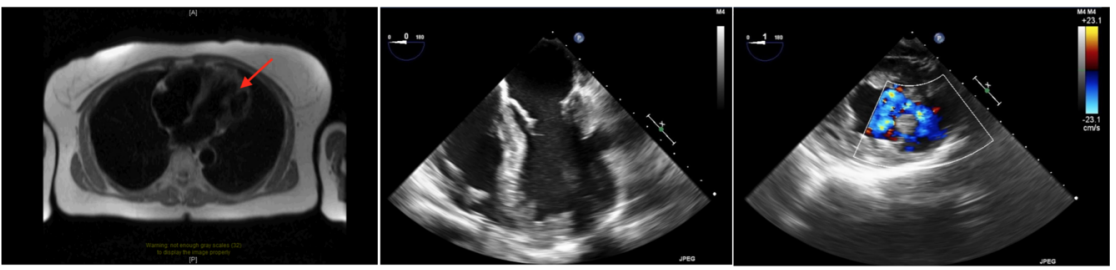

Figure 2: Intra-operative transesophageal echocardiogram and pre-operative magnetic resonance imaging 